\title{
2x2 Grid Array Design with E-shaped Microstrip Elements
}

\author{
Juhi K. Baruah \\ Department of Electronics and Communication Engineering \\ Gauhati University \\ Guwahati,India \\ baruahjuhikangkana@gmail.com \\ Kandarpa Kumar Sarma \\ Department of Electronics and Communication Engineering \\ Gauhati University \\ Guwahati,India \\ kandarpaks@gauhati.ac.in
}

\author{
Sivaranjan Goswami \\ Department of Electronics and Communication Engineering \\ Gauhati University \\ Guwahati,India \\ sivgos@gmail.com \\ Nikos E. Mastorakis \\ Department of Industrial Engineering \\ Technical University of Sofia \\ Sofia, Bulgaria \\ mastor@tu-sofia.bg
}

Received: March 6, 2021. Revised: August 10, 2021. Accepted: September 6, 2021. Published: September 8, 2021.

\begin{abstract}
The paper proposes a work of four element in a $2 \times 2$ grid fashioned with $E$-shaped microstrip patch antenna with corporate fed .The paper compares the proposed design with four elements with a single element and a 2 element array design.All the three antenna designs use $\mathbf{E}$ shaped microstrip patch as an element. The design of the grid is achieved through the design of a single element, the design of a $1 \times 2$ array and finally the design of the $2 \times 2$ grid on an FR4 epoxy substrate of thickness $1.5 \mathrm{~mm}$. A corporate feed network of microstrip lines is used to excite the array. The performance of each stage is studied in terms of the return loss parameter, the far field gain, and the beamwidths are observed in each case from simulation results. The resonant frequency in each case is $3.8 \mathrm{GHz}$. Through comparision of simulation results the paper shows that as the number of elements is increased, the beam-width reduces. In other words, the directivity is increased. Further, it is also observed that the gain and bandwidth is the minimum for the single patch, followed by that of the $1 \times 2$ array and the maximum for the $2 \times 2$ grid. Thus,it is ssen that the proposed four element antenna with corporate feeding performs better as compared to antennas with either only single patch element or two element array. The construction of the grid leads to increase in gain, bandwidth and directivity of the antenna.
\end{abstract}

Keywords- Microstrip patch antenna, 3.8GHz, stub matching, $2 \times 2$ grid pattern, Antenna gain

\section{INTRODUCTION}

Progress in the field of technology has become inexorable with the beginning of Industrial revolution.with the recent implementation of $5^{\text {th }}$ generation (5G) of mobile communication in many countries the world is now moving towards the future generation.

Microstrip patch antennas have received significant attention worldwide in the past few decades because of its small size, low profile, light weight, low production cost and other aspects of the advantage [1][2] which are suitable for commercial wireless communication . Today it is widely being used in mobile communications, satellite communications, radar, and other various fields [3]. The performance of an antenna can be enhanced in terms of different parameters by constructing an array of microstrip antennas. Microstrip antennas can have different shapes such as rectangular,circular,triangular etc. Modification in the shape of patch antennas has helped to aquire desirable antenna performance. Also these supports various feeding techniques by help of which antennas can be turn into arrays to achieve desired antenna requirements. Along with that it can support high packaging density. Patch antenna manufacturing cost is also very low . Till now various array forms of microstrip patch antennas have been designed [4][7] for various applications such as GSM, radar etc. An array can be designed as a one dimensional array consisting of antenna elements along a line or it can be designed in a grid pattern by placing equal numbers of patch in row and coloumn wise as in [3][4]. In addition to the basic advantages of the microstrip patch antenna, the grid pattern antenna has the advantages of high gain, high bandwidth, narrow focused beam, low sidelobe and radiation characteristics [9].

Hinging to these reasons patch antennas have come out as an strong option for millimeter wave application.Thus $5 \mathrm{~g}$ antennas have been developing with patch element technology.

In this paper a $2 \times 2$ microstrip patch grid array antenna is designed at a frequency of $3.8 \mathrm{GHz}$. The antenna is having an element of E-shaped patch, as it has a larger bandwidth as compared to a rectangular patch [10]. The antenna is designed with a single patch element first and then it is transformed to a $1 \times 2$ element array with corporate feeding.this corporate feeding technique is very popularly used for microstrip array antennas. In this technique equal amount of power gets splitted at each junction of the microstrip patch array antenna to maintain uniform distribution. Finally from the designed $1 \times 2$ element array, a $2 \times 2$ grid of microstrip patch antennas is derived out by combining the two $1 \times 2$ element arrays. Initially, a quarter wavelength transformer is used for impedance matching in the $1 \times 2$ element array. Later a stub matching technique is introduced to design the $2 \times 2$ grid with four element and to ensure that the return loss is below $-15 \mathrm{~dB}$.

Stub matching can be use to match load to the transmission line. These are basically consisting of shortened or opened segments of the line connected either in series or in parallel with the line at a proper distance from the load. 
The design procedure of all the three design configurations: the single patch element, the $1 \times 2$ element array, and the $2 \times 2$ grid array element is discussed in section II. Section III includes the simulation and measurement results of all the three design configurations and discussions of the work in each case. Finally, the conclusions based on the results obtained are made in section IV.

W

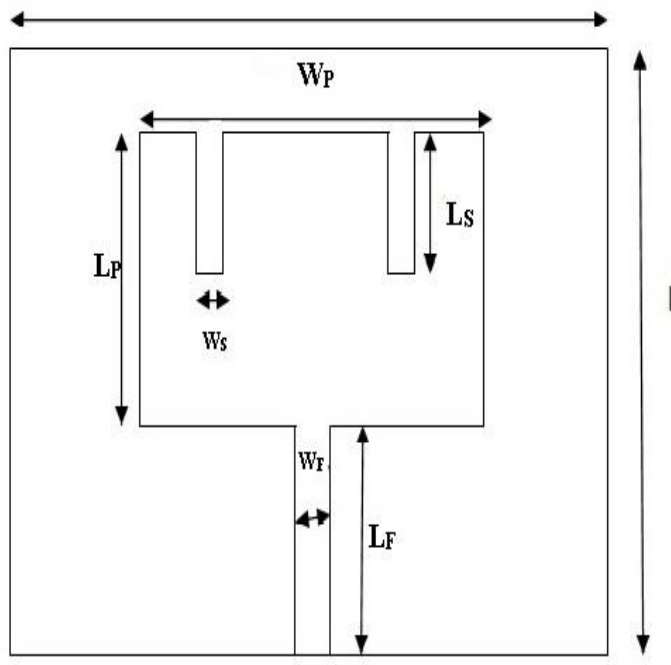

Fig.1 Micro strip single patch E- shaped antenna

\section{DESIGN PROCEDURE}

The design procedure involves the design of the single patch antenna element followed by the design of a $1 \times 2$ element array and finally a $2 \times 2$ grid of four microstrip patch element antennas. Here, FR4 epoxy substrate with a dielectric constant, $\varepsilon_{\mathrm{r}}$ of 4.4 and a thickness of $1.5 \mathrm{~mm}$ is used for the designs. The details of these design steps are discussed as follows.

\section{A. Design of a single patch E-shaped antenna}

First, the single patch element that is to be implemented into arrays with feeding was designed. For this single patch element design the topology of the E-shaped patch antenna element and its various geometrical parameters are shown in Figure 1. The tentative length and width of the patch were calculated as $23 \mathrm{~mm}$ and $30 \mathrm{~mm}$ respectively with the operating frequency. However to achieve the desired results the values were optimized to the tabulated value. Also to obtain an E shape out of the rectangular patch two small slots of sizes $0.8 \mathrm{~mm}$ length and $0.5 \mathrm{~mm}$ width are cut out.

The final shape is then obtained and the dimensions are experimentally tuned with Ansys HFSS version 13 to obtain resonance at $3.8 \mathrm{GHz}$ along with a return loss of -16 dB. The overall dimensions of the single patch element antenna design are shown in Table 1.
In the design a simple feedline of width $2.868 \mathrm{~mm}$ is also used to fed the patch element as shown in figure 1 .

TABLE I. DIMENSIONS OF SINGLE PATCH ANTENNA

\begin{tabular}{|l|l|}
\hline Specifications & Dimensions (in mm) \\
\hline Length of the substrate, $\mathrm{L}$ & 60 \\
\hline Width of the substrate, $\mathrm{W}$ & 60 \\
\hline Length of patch, $\mathrm{L}_{\mathrm{P}}$ & 18.4 \\
\hline Width of patch, $\mathrm{W}_{\mathrm{P}}$ & 25 \\
\hline Feed width, $\mathrm{W}_{\mathrm{F}}$ & 2.868 \\
\hline Slot length, Ls & 0.8 \\
\hline Slot width, $\mathrm{W}_{\mathrm{S}}$ & 0.5 \\
\hline
\end{tabular}

\section{B. $1 \times 2$ Patch Antenna design}

After designing the single patch element antenna with required performance the patch elements were combined and the $1 \times 2$ array is designed using the E-shaped patch antenna as elements. The dimensions of the patch element in this design are kept the same so that the array also resonates at $3.8 \mathrm{GHz}$ similar to the single patch element design . Here in this design a corporate feeding network is used to fed the elements. These patch elements are fed in parallel using microstrip transmission lines. These microstrip transmission lines are divided into two branches in order to match the impedance of patch elements. The power division is accomplished by using quarter wavelength transformer [11] with approximately $70.71 \mathrm{ohm}$ to match $100 \mathrm{ohm}$ to 50 ohm as in [6]. The space between the two patch elements kept here is one fourth of the wavelength $(\lambda / 4)$ that is $19.723 \mathrm{~mm}$. Fig. 2 shows the design view of the specified $1 \times 2$ array antenna design.

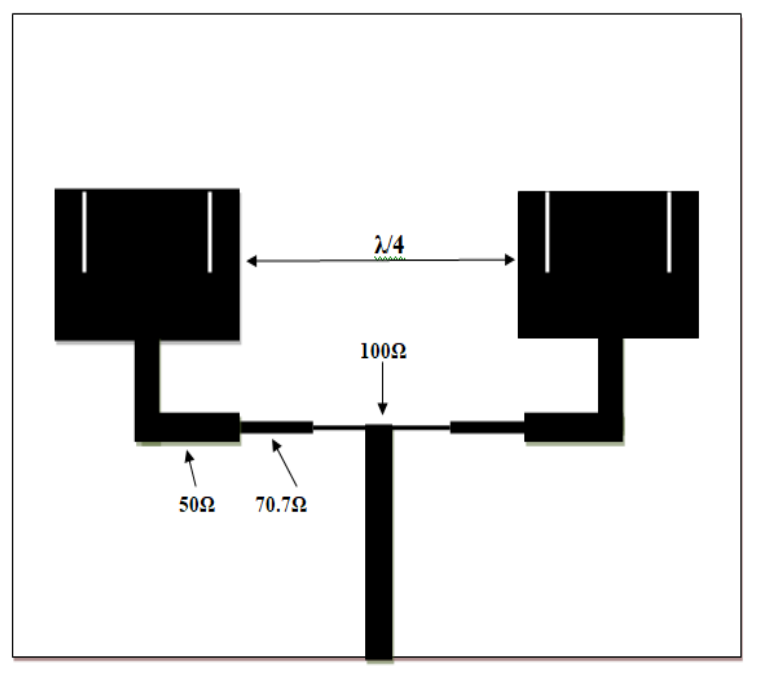

Fig.2. Layout of $1 \times 2$ patch antenna

\section{C. $2 \times 2$ Grid micro strip patch design}

After successfully desining the $1 \times 2$ array,the four patch elements were combined to form a grid array pattern. In this design of $2 \times 2$ micro strip patch grid array antenna, the parameters of each single patch elements were kept 
unchanged wheras only the overall antenna size was changed. All the patch elements were arranged in a grid fashioned array separated by a distance equal to approximately $\lambda / 4$. The feed line splits into two branches similar to that in case of the $1 \times 2$ array. Each branch further splits into two sub-branches each of which excites one Eshaped patch element antenna as in [7].

Quarter wavelength transformer technique is used in each split to ensure proper impedance matching.

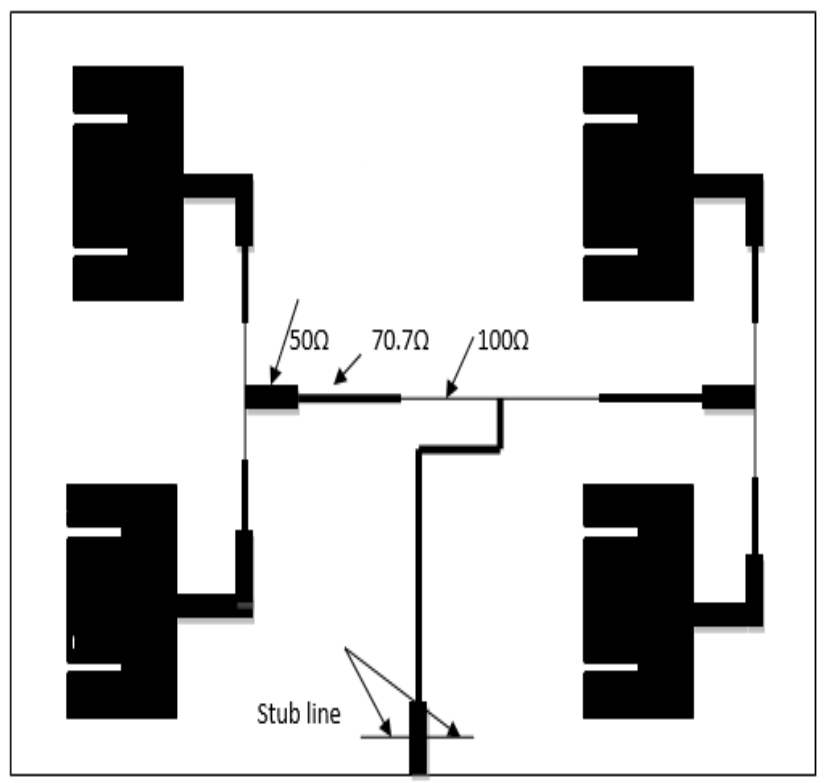

Fig.3.Layout of $2 \times 2$ Micro strip patch Grid antenna

Although quarter wavelength transformers are used at each split of the corporate feeding network, experimentally it was observed that the impedance was not perfectly matched and the return loss was above - $10 \mathrm{~dB}$ which was not the desirable performance. So in order to further improve the impedance matching, stub lines are used just near the feed position of the corporate network as shown in Figure 3. Each of the two stub lines has a width of $0.5 \mathrm{~mm}$ and length of 5 mm. A similar approach for impedance matching was reported in [5].

\section{RESULTS AND DISCUSSION}

The single patch element antenna, the $1 \times 2$ elementarray and the $2 \times 2$ element grid array are designed and simulated in HFSS 13 to analyze the results. Further the final $2 \times 2$ element grid array antenna design is fabricated on a copperFR4 epoxy PCB and the performance is measured using a vector network analyzer (VNA) and a radiation pattern measurement setup. The simulation and measurement results are discussed here in this section.

\section{A. Analysis of Resonant Frequency and Return Loss}

The frequency reponse of the return loss parameter (S11), obtained for the single patch antenna from simulation results, is shown in Fig. 4. It is observed that the antenna has a resoant frequency of $3.8 \mathrm{GHz}$ and a bandwidth of 140 $\mathrm{MHz}$. The return loss obtained at $3.8 \mathrm{GHz}$ is $-15 \mathrm{~dB}$.

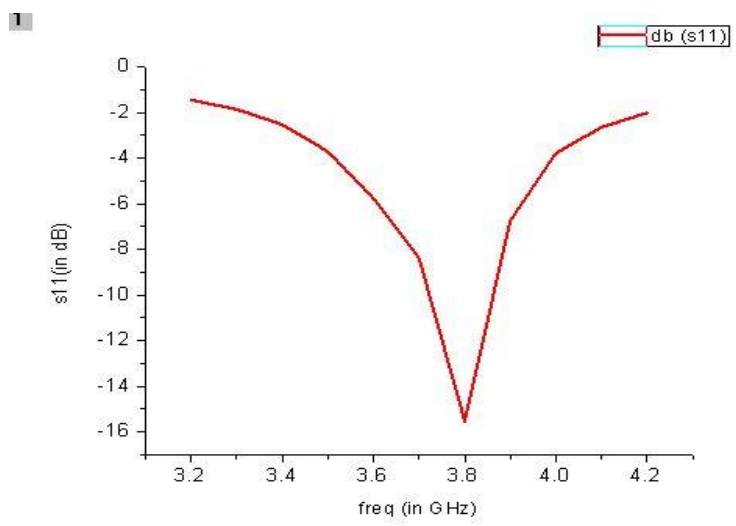

Fig.4. Return loss plot for E-shaped single patch antenna

In case of $1 \times 2$ array, the $\mathrm{S} 11$ vs. frequency plot is shown in Fig. 5. The resonating at desired frequency of this structure is also obtained at $3.8 \mathrm{GHz}$ with a bandwidth of $145 \mathrm{MHz}$. The return loss obtained at the resonating frequency is -25 $\mathrm{dB}$.

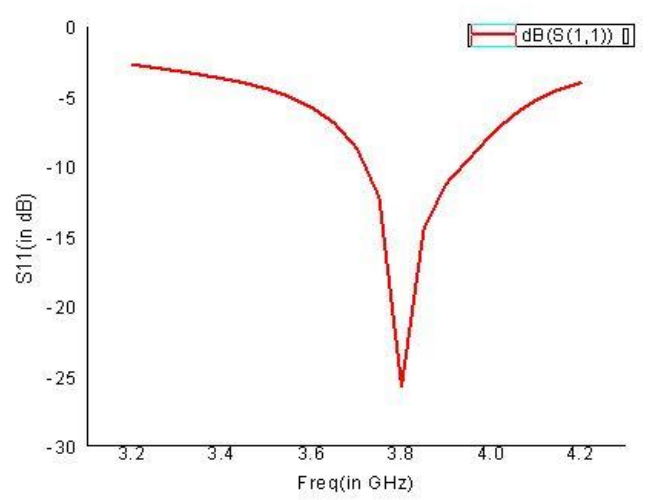

Fig.5. Comparison plot of s11 for single patch and $1 \times 2$ antenna

Figure 6 shows the return loss for our desired $2 \times 2$ grid antenna. From the plot it can be seen that the antenna is delivering the minimum return loss of $-16.5 \mathrm{~dB}$, with a bandwidth of $160 \mathrm{MHz}$.

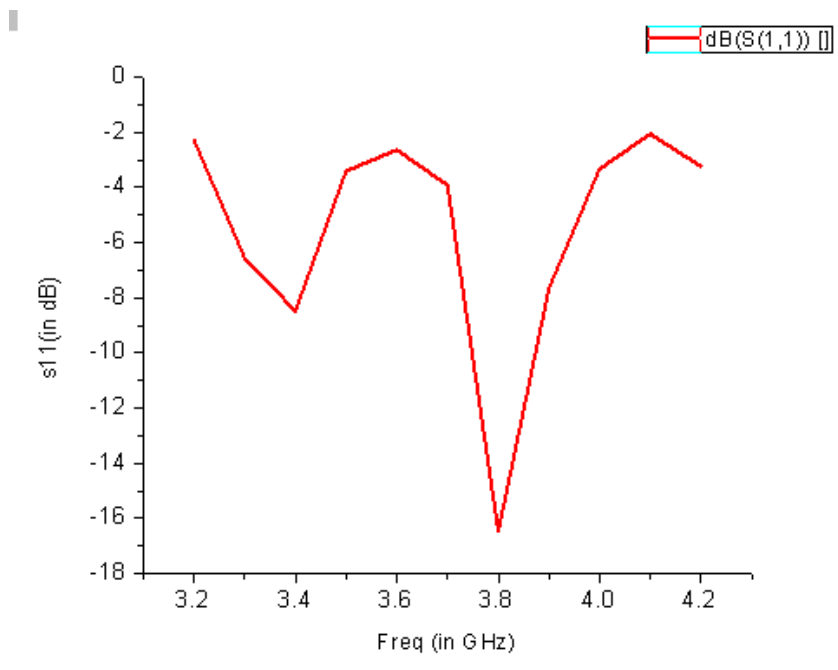


Fig.6 Return loss plot for E-shaped 2x 2 grid pattern antenna

Basically single patch elements are combined to form the final array antenna.Thus in such cases return loss is similar to that of single patch .however the final array antenna is showing a better result of return loss as compared to the single patch element design.

\section{B.Antenna Gain and Radiation Pattern}

Obtaining enhanced gain and narrow beamwidth is the key advantage of constructing arrays from individual antenna elements. The radiation patterns of the far field gain of each of the single patch element antenna, $1 \times 2$ element array antenna, and the $2 \times 2$ grid array antenna design are obtained from simulation results and compared.

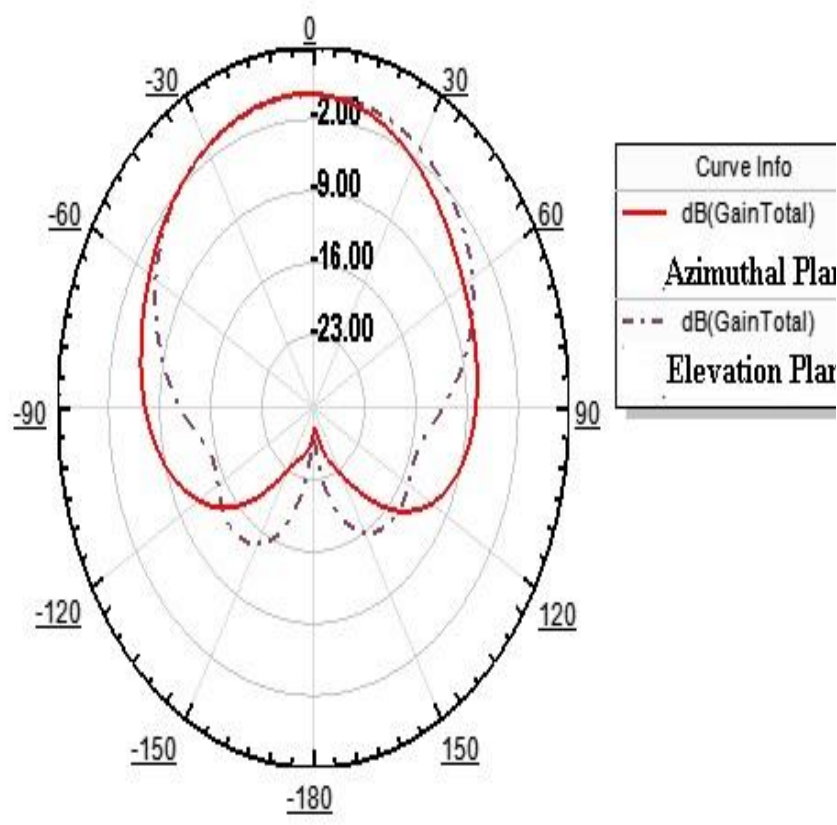

Fig.7. Radiation pattern of single patch antenna

Fig. 7, 8 and 9 show the far field radtion pattern in the azimuthal plane and elevation plane of the single patch element antenna, the $1 \times 2$ array antenna and the $2 \times 2$ grid array antenna respectively. It is observed that in fig 7 , the single patch has a peak gain of $2.3 \mathrm{dBi}$ along the direction of the major lobe at frequency $3.8 \mathrm{Ghz}$.The beamwidth is slightly less narrower in this case.

In fig 8 the radiation pattern plot of $1 \times 2$ array is showing a peak gain of $3.4 \mathrm{dBi}$ along the major lobe.The total gain in $\mathrm{dB}$ in both the azimuthal and elevation plane has been shown. However the beamwidth in the elevation plane is narrower in this case than the single element but in azimuthal plane the beamwidth is less narrower.

In fig 9.the radiation pattern plot of the $2 \times 2$ grid array antenna is showing a peak gain of $6.5 \mathrm{dBi}$ which is way more than both of the previously designed single patch element antenna design and $1 \times 2$ element array antenna design Thus, we can come up with the fact that the gain is enhanced as the number of elements in an array is increased or more numbers of antenna elements were added.The gain obtained in this case is more than double to that of the single patch element and nearly double to that of the $1 \times 2$ element array.

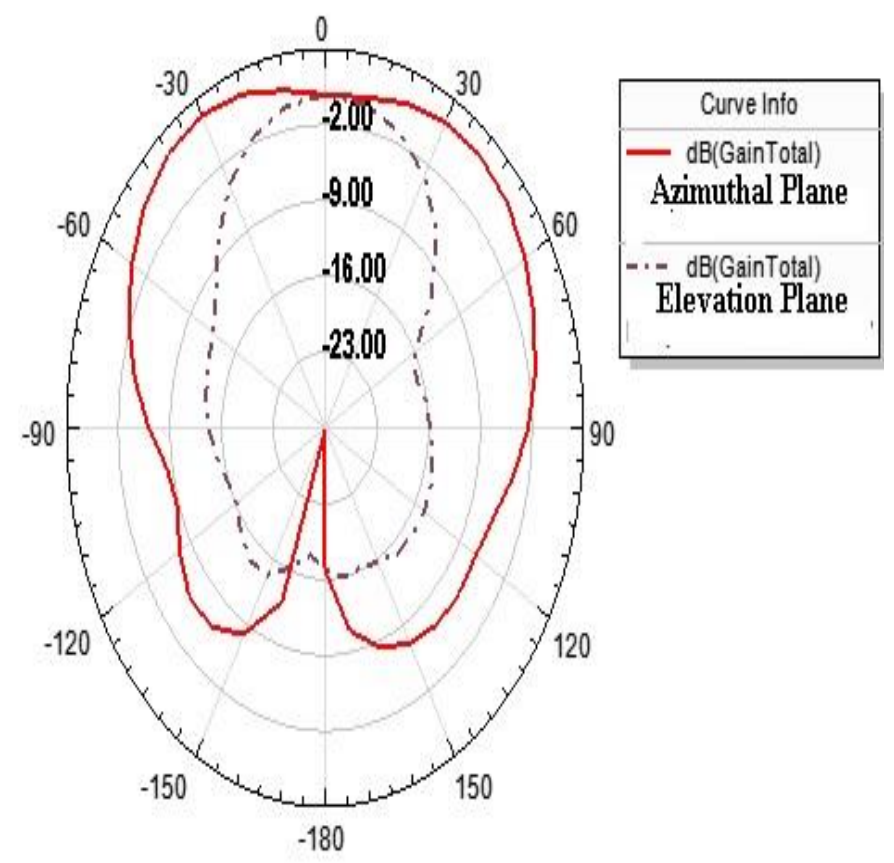

Fig.8. Radiation pattern of $1 \times 2$ patch antenna

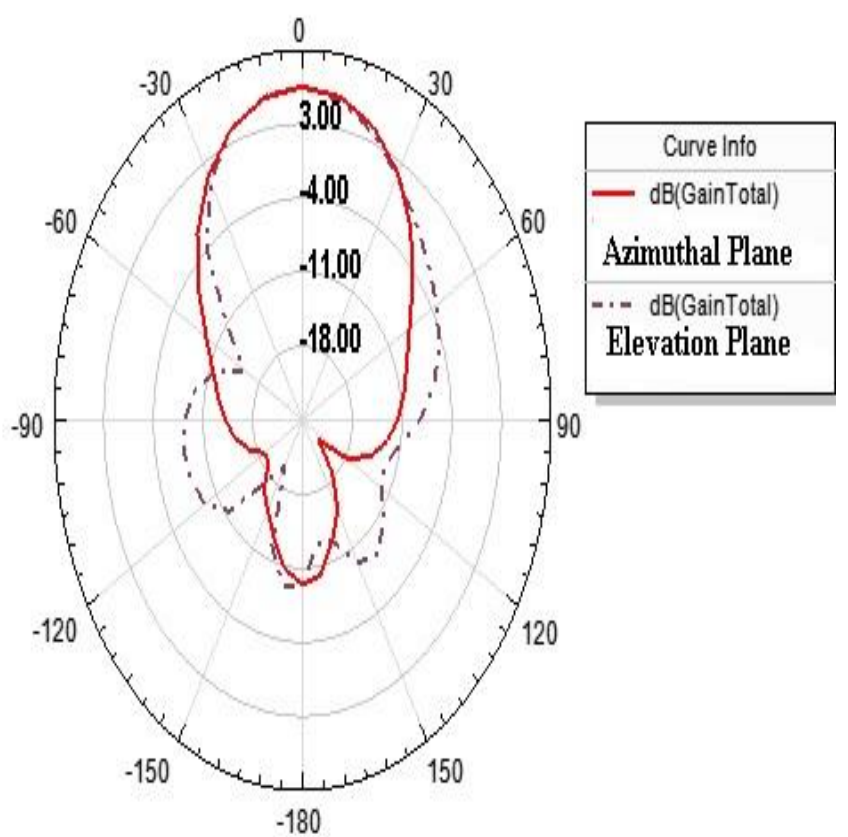

Fig.9. Radiation pattern of $2 \times 2$ Grid micro strip antenna 
Comparing the radiation patterns in Fig. 7 and 8, it is observed that although the $1 \times 2$ array has a less narrower beamwidth but when compared to the radiation pattern of the single patch element antenna. It has a more narrower beamwidth in the elevation or $\mathrm{E}$ plane. However, in the azimuthal plane, the presence of a single broadside lobe is not observed. Again when radiation plot of $2 \times 2$ grid array antenna in Fig. 9 is compared with radiation plot of single patch element antenna in Fig. 7 it is observed that the major lobe of the $2 \times 2$ grid is significantly narrow in both azimuthal and elevation plane as compared to the single antenna element.Also it has narrower beamwidthin comparision to the $1 \times 2$ element array in both the azimuthal plane and the elevation plane.However some minor back lobes are observed in both the cases of the $1 \times 2$ element array antenna design and also in $2 \times 2$ grid array antenna design.

\section{Fabrication and Measurement Results}

The final design of $2 \times 2$ grid array antenna is fabricated on a copper-FR4 epoxy substrate with a thickness of $1.5 \mathrm{~mm}$ and a dielectric constant, $\varepsilon_{\mathrm{r}}$ of 4.4 as shown in Fig. 10. The frequency vs. return loss (S11) parameter plot for the grid array antenna was obtained from Vector Network Analyser (VNA) for the fabricated antenna. The plot so obtained is compared with the simulation results for the same antenna and is shown in Fig. 11.Here, the return loss is plot for both the simulated result and the measured result.

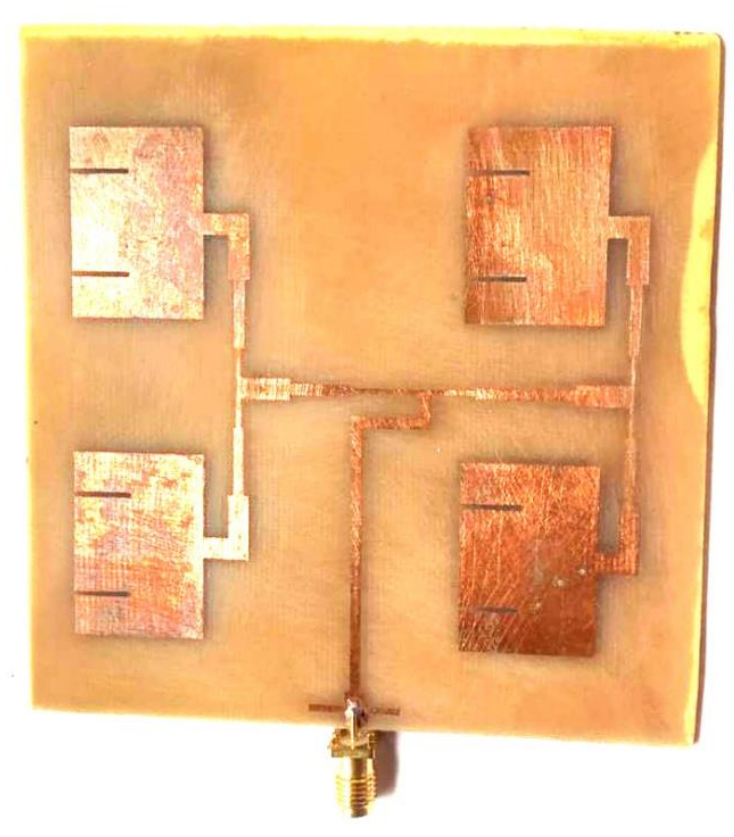

Fig. 10: The fabricated $2 \times 2$ grid array with E-shaped patch antenna elements

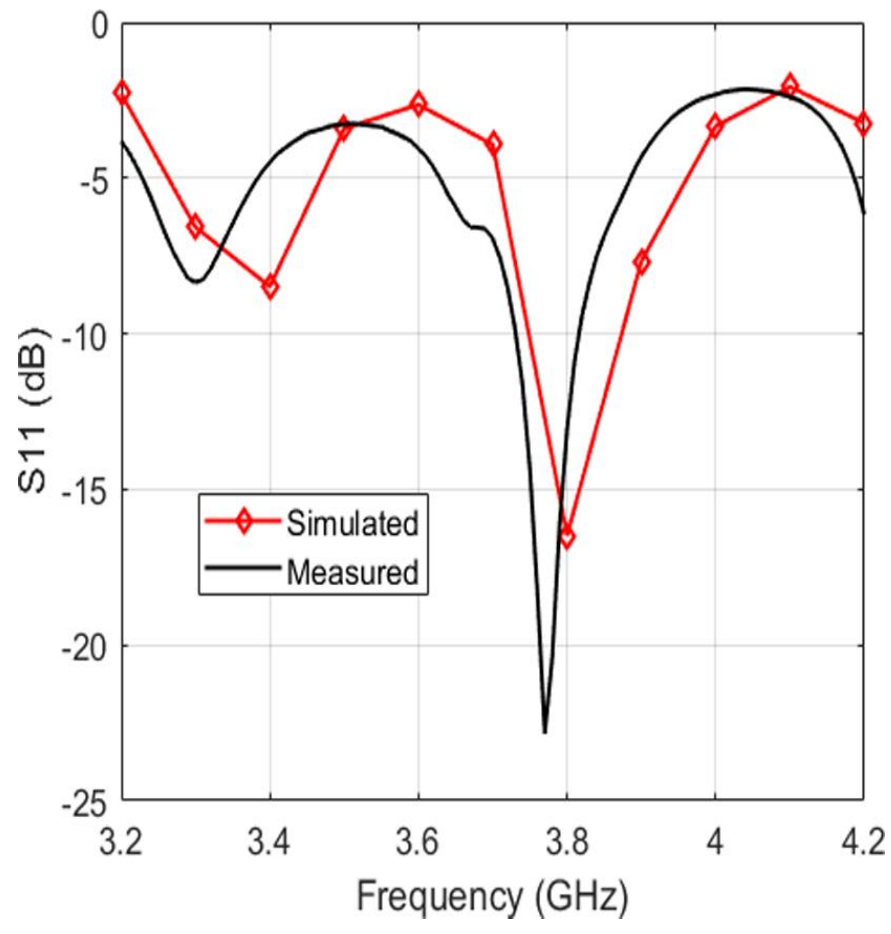

Fig. 11 Comparison of the simulated and measured S11 parameter plot for the $2 \times 2$ grid antenna

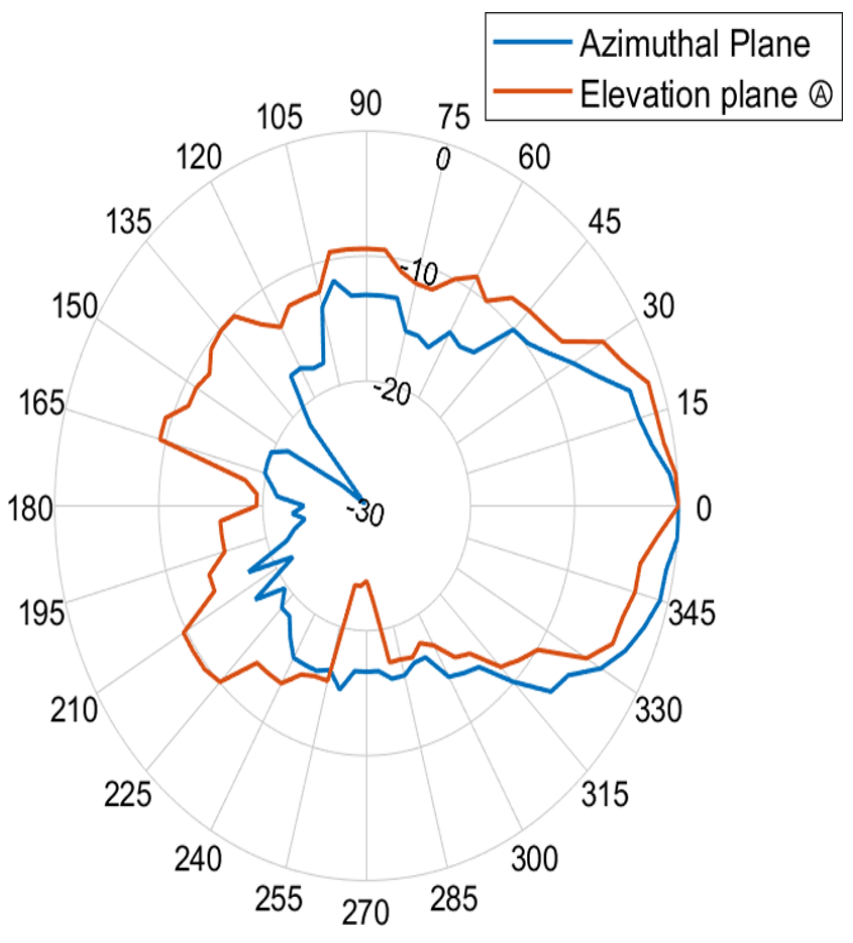

Fig. 12 Measured normalized radiation pattern of the $2 \times 2$ grid antenna in the azimuthal plane and the elevation plane

The measured far field radiation pattern of the antenna in the azimuthal plane and the elevation plane is shown in Fig. 12. 
It is observed from the measurement results that the fabricated antenna resonates at a frequency of $3.77 \mathrm{GHz}$ which is very close to the operating frequency $3.8 \mathrm{GHz}$. Also the radiation pattern of the fabricated grid array antenna is almost similar to that of the simulation results.

\section{B. Discussion}

From the analysis of the resonant frequency and also the return loss as presented in the Section III (A), it is observed that the bandwidth of the antenna with $1 \times 2$ array element is more than that of the single patch element antenna by a factor of around $5 \mathrm{MHz}$. This bandwidth is again further increased by about $15 \mathrm{MHz}$ when the $2 \times 2$ grid array antenna is constructed as compared to that of the $1 \times 2$ element array antenna. The $2 \times 2$ grid array antenna has a bandwidth of $160 \mathrm{MHz}$ which is significantly large in comparison to that of a single patch element antenna .

In each of the design case, a directional radiation pattern is obtained either for single patch element antenna or $1 \times 2$ element array antenna or $2 \times 2$ grid array antenna. It is also seen that the far field peak gain increases as the number of elements is more as discussed in Section III (B).

Table II presents the comparision of antenna designssingle patch, $1 \times 2$ element array and $2 \times 2$ grid array antenna in terms of Bandwidth (in $\mathrm{MHz}$ ), Gain (in $\mathrm{dBi}$ ) and radiation pattern.

TABLE II. COMPARISON TABLE OF RESULTS

\begin{tabular}{lccc}
$\begin{array}{l}\text { Antenna } \\
\text { specifications }\end{array}$ & \multicolumn{3}{c}{ Antenna configuration type } \\
& Single patch & $\mathbf{1 \times 2}$ array & $\mathbf{2 \times 2}$ grid \\
$\begin{array}{l}\text { Bandwidth } \\
\text { (in MHz) }\end{array}$ & 140 & 145 & 160 \\
Gain (in dBi ) & 2.3 & 3.4 & 6.5
\end{tabular}

Radiation pattern Directional Directional Directional

.From the above comparision table it is clear that the $2 \times 2$ grid array antenna outperforms the $1 \times 2$ element array antenna and single patch element antenna in terms of gain bandwidth and beamwidth, aking it more desirable for future communication applications.

\section{IV.CONCLUSIONS}

Designs of single patch element antenna, $1 \times 2$ element array antenna and $2 \times 2$ grid array antenna are presented in this paper.Further feeding techniques were merged like coporate fed with quarter wavelength transformer technique. Also the stub matching technique was applied on the design to obtain a more perfect impedance match. This paper presented the proposed antenna design and further fabricated and compared the measured results with the simulation results. The proposed design and the experimental results show that by constructing a $2 \times 2$ grid of E-shaped patch antenna elements, the gain, the bandwidth and the directivity can be enhanced. The $2 \times 2$ grid yields a larger gain and bandwidth compared to a single patch antenna and a one-dimensional $1 \times 2$ array. From the comparative analysis of the radiation paterns as presented in Section II(B), it may be concluded that a one dimensional array of microstrip antennas enhance the directivity of the antenna only in the azimuthal plane. The proposed $2 \times 2$ grid of the antenna increases the directivity in both the azimuthal plane and the elevation plane. This is an additional advantage of a 2-dimensional grid of microstrip antennas. Overall from the results it is proved that propose antenna outperforms the single patch and also the $1 \times 2$ element array antenna making it best for the future wireless communication like smart radar antennas or in the field of IoT.

\section{ACKNOWLEDGMENT}

The author expresses a heartiest gratitude to MHRD TEQIP III for their support .

\section{REFERENCES}

[1] R. A. Abd-Alhameed1, D. Zhou1, c. H. See1, and P. S. Excel, "A Wire Grid Adaptive-Meshing Program forMicrostrip-Patch Antenna Designs sing aGenetic Algorithm", IEEE Antennas and Propagation Magazine Vol. 51, No.1, February 2009.

[2] K.Shrikar ,L.S Vinodh, B .Ramesh and K.P Vinay, "1x2 triangular shape Microstrip patch antenna array for WLAN applications with dgs structure," International journal of Innovative research in computer and Communication Engineering, vol. III ,issue III , March 2015, ISSN. 2320-9801

[3] S.P.Sinha ,M.Kumar, and J Gupta, "Design of $2 \times 2$ Shaped Rectengular Micro strip Array Antenna for GSM Application," International journal of Scientific and Engineering research, vol 6 ,issue 5, May 2015, ISSN. 2229-5518

[4] J.George and B.Lethakumari, "Design and optimization of $2 \times 2$ corporate - series fed micro strip antenna array," International Journal of Engineering \& Technology, 7 (1.9), 2018, 84-87

[5] A. Slowik, M. Czyzewski and B. Slesicki, "The use of shunt-stubs in corporate-feeding network for S-band planar antenna array, " 2018 22nd International Microwave and Radar Conference (MIKON), Poznan, 2018, pp.360-362. doi: 10.23919/MIKON.2018 .8405225

[6] H. M. M. Makkawi, "Performance evaluation of $1 \times 2$ patch antenna array based on power divider characterises," 2016 Conference of Basic Sciences and Engineering Studies (SGCAC), Khartoum, 2016, pp.6-12. doi: 10.1109/SGCAC.2016.7457998

[7] M. S. R Mohd Shah, M. Z. A Abdul Aziz and M. K. Suaidi, “Dual Linearly Polarized Microstrip Array Antenna', as a chapter in Trends in Telecommunications Technologies, Christos J Bouras (Ed.), pp. 367-388, 2010.

[8] B.T.P. Madhav, H. Khan, and V.G.N.S Prasad, "Microstrip 2x2 antenna on k15 liquid crystal substrate," International journal of applied engineering and research, vol. 6,number 9, pp. 1099$11041,2011$.

[9] Mei Sun, Yue Ping Zhang, Duixian Liu, Kai Meng Chua, and Lai Lai Wai, A Ball Grid Array Package With a Microstrip GridArray Antenna for a Single-Chip 60-GHz Receiver, IEEE Transactions on Antennas and Propagation, VOL. 59, NO. 6, JUNE 2011

[10] B.Akhilesh, G.Karthikeya, M.Sujatha, "Design of E-Shape Microstrip Patch Antenna for UltraWideband Applications", IOSR Journal of Electronics and Communication Engineering,vol 9, issue 2,pp 14-18, Apr 2014.

[11] D. M. Pozar, Microwave engineering. John Wiley \& Sons, 2009.

\section{Creative Commons Attribution License 4.0 (Attribution 4.0 International, CC BY 4.0)}

This article is published under the terms of the Creative Commons Attribution License 4.0

https://creativecommons.org/licenses/by/4.0/deed.en_US 